\title{
An Overview on Interference Management in 3GPP LTE- Advanced Heterogeneous Networks
}

\author{
Md. Shipon Ali \\ Technology Division, Grameenphone Ltd., Dhaka, Bangladesh \\ shipon@grameenphone.com
}

\begin{abstract}
The increasing demand for mobile data traffic brings new challenges on cellular networks in terms of network capacity and increased data throughput. In order to fulfill these demands, Heterogeneous Networks (HetNets) has been considered as a promising solution. On the other hand, Inter-Cell Interference Coordination (ICIC) is a big challenge toward HetNet deployments; in particular users in edge area of low power small cell are mostly vulnerable to stronger interference signals from high power macro cell. To resolve ICIC problem, 3GPP released some technologies based on TDM resource partitioning, FDM resource allocation along with power control, and still continue to find out the more suitable solutions. However, to date, a common platform of all possible solutions, particularly in TDM and FDM based, is not available to readers. In this context, this paper provides a holistic overview of ICIC strategies in this emerging wireless system. Towards this objective, the evolution of inter-cell interference management techniques is thoroughly investigated from TDM based enhanced inter-cell interference coordination (eICIC) techniques to more advanced Coordinated Multi-Point (CoMP) transmission/reception, while comparing and contrasting their common features and differences. Finally, Carrier Aggregation (CA) is explored in detail as an advanced and challenging mechanism in order to have an efficient resource allocation and intercell interference mitigation in LTE-Advanced HetNet environments.
\end{abstract}

Keyword-LTE-Advanced, HetNets, eICIC, FeICIC, Carrier Aggregation, CoMP.

\section{Introduction}

The rapid growth of mobile users demands the fast development of wireless communication technology. According to Ericson [1], the subscriptions of world-wide mobile broadband user are expected to grow to 3.5 billion by 2015, which is around two times higher than the current trends. In addition, according to Cisco [2], global mobile data traffic is expected to grow up to 11.2 Exabyte per month by 2017. However, in order to meet these increasing requirements of the mobile data traffic, the 3rd Generation Partnership Project (3GPP) Release 10 has introduced advanced features of LTE, referred as LTE-Advanced, which includes the Carrier Aggregation (CA), Heterogeneous Networks (HetNets), Cooperative Multi-Point (CoMP) communication, enhanced MIMO, enhanced ICIC. This enhanced system is referred as the LTE-Advanced (LTE-A) system.

HetNets can be realized by overlaying low power small cells, e.g. Pico, Femto, RRH etc., within high power macro-cell coverage area, thus achieve greater cell splitting gains. Though HetNets provides significant capacity gain for mobile data traffic, it also brings heavy interference particularly at user end. In addition, to get more advantages from HetNets a technique called Cell Range Expansion (CRE) of low power small cell is applied which offloads more traffic from macro-cell to small cells, but results more interference scenarios. Moreover, MU-MIMO applications further enlarge the interference zone, inter-UE interference at base station end. However, from beginning of LTE standardization 3GPP have been keeping high concentration on ICIC. While Release 
8/9 ICIC works well for homogeneous networks, it falls short of providing significant gain in a heterogeneous network (HetNet) architecture because it limits range expansion as it applies only to data channels and not to control channels where interference can remain significant. Later, Release 10 introduced enhanced Inter-cell Interference Coordination (eICIC) techniques based on TDM resource partitioning which allows a number of zero/reduced power subframes of macro-cell those are likely to make interfere with the UEs associated to small cells. After then, Release 11 revealed the Furtherenhanced ICIC (FeICIC) technique based on transmitter/receiver action which demonstrates the effective solution that was addressed in Release 10, but also bring some new challenges. On the other hand, Carrier Aggregation (CA) based HetNets has been considered as a promising solution of inter-cell interference problem.

The rest of the paper is organized as follows: section 2 presents the motivation towards this work and also mentions related major works that have been previously performed in this regard. A basic LTE/LTE-Advanced principle is discussed in section 3. Section 4 and 5 briefly presents heterogeneous networks and cell range expansion technique, respectively. The detail description of various ICIC techniques including TDM based solution, FDM based solution and CoMP implementations are presented in section 6 . Finally, section 7 contains the concluding part along the potential challenges of implementations.

\section{Motivation And Related Works}

Recently, the concept of HetNets has attracted a lot of interest to optimize the performance of the radio network. This is due to the fact that the spectral efficiency of current systems like WCDMA and LTE is approaching theoretical boundaries [14], thus additional spectrum and advanced network topology are the main resource to improve the network capacity. But, spectrum has been a scarce resource in the past few years; thus, the main approach to enhance the performance is to improve the network topology.

Regarding the utilization of HetNets as a way to improve network capacity and performance in relation to traditional homogeneous network are discussed in [7]-[16]. In [7], authors discuss a high-level overview of LTE heterogeneous network in terms of air interface, network nodes, spectrum allocation along with other mechanisms for HetNets deployments. In [11], the authors described the interference coordination schemes and technical challenges that arise in heterogeneous networks highlighting the impact of interference in deployments consisting of cells of different size. Also, authors in [11] described several techniques enabling the move from macro-only to HetNets including range extension and enhanced inter-cell interference management techniques over HetNets. However, in context of ICIC management in HetNets, 3GPP Release 10 introduced $\mathrm{ABS} /$ based eICIC which is an efficient way to enhance network performance, in particular for cell-edge/expansion area UEs. In addition, authors in [11], [14], [15] addressed downlink co-channel interference in context of ABS-based eICIC at different deployment scenarios, where [11] and [12] deals with macro-pico approach and [15] studies both of the macro-pico and macro-femto approaches. The both studies have been evaluated in different network architectures, namely centralized and distributed architecture, and suggest a considerable performance gain by means of HetNets.

On the other hand, interference management schemes based on FDM and scheduling algorithm are presented in [9], [18]. In [9] authors proposed a dynamic interference coordination scheme based on the combination of soft frequency reuse and Graph-based resource allocation on macro-pico scenario. Authors in [18] discussed the key terms of scheduling through interference avoidance/coordination to mitigate interference in a multi-cell environment. Carrier Aggregation (CA) based radio resource allocation and inter-cell interference mitigation has been extensively described in [15]-[17]. In [16], multi-site CA has been introduced which effectively allows multiple uplink in support of 
Timing Advance (TA) enhanced uplink power control, and resulting avoid the interference by ensuring coordination among the transmission points. However, to the best of my knowledge, a holistic survey on inter-cell interference coordination in LTEAdvanced HetNets with particular focus on TDM based eICIC, FeICIC and carrier aggregation is not currently available. Therefore, motivated by the urgency to bring all the possible solutions of ICIC in LTE-Advanced HetNets into a single platform, this article is prepared.

\section{LTE/LTE-Advanced Overview}

In early 2009, 3GPP published LTE standard in targets of 4G cellular network which is referred as Release 8. LTE is based on the OFDMA for downlink and Single-Carrier FDMA (SC-FDMA) for uplink transmission. Compared to other existing technology LTE offered higher peak data rates mainly due to unique features, larger system bandwidth and higher order MIMO technology. However, as other cellular technologies LTE is continuously worked on in terms of improvements and initial enhancements were included in Release 9, followed by more significant improvements in Release 10 which is known as LTE-Advanced [3,5]. Table 1 shows the spectral efficiency targets of LTE, LTE-Advanced and ITU requirements for IMT-Advanced.

Table 1: Spectral Performance Target of LTE, LTE-Advanced and IMTAdvanced Requirement

\begin{tabular}{|c|c|c|c|c|}
\hline Item & $\begin{array}{c}\text { Sub- } \\
\text { category }\end{array}$ & LTE (Rel 8) & $\begin{array}{c}\text { LTE- } \\
\text { Advanced }\end{array}$ & $\begin{array}{c}\text { IMT- } \\
\text { Advanced }\end{array}$ \\
\hline \multirow{2}{*}{$\begin{array}{l}\text { Peak spectral efficiency } \\
(\mathrm{b} / \mathrm{s} / \mathrm{Hz})\end{array}$} & Downlink & $\begin{array}{c}16.3 \\
(4 \times 4 \text { MIMO })\end{array}$ & $\begin{array}{c}30 \\
(8 \times 8 \text { MIMO) }\end{array}$ & $\begin{array}{c}15 \\
(4 \times 4 \text { MIMO })\end{array}$ \\
\hline & Uplink & $\begin{array}{c}4.32 \text { (64- } \\
\text { QAM SISO) }\end{array}$ & $\begin{array}{c}15 \\
(4 \times 4 \text { MIMO) }\end{array}$ & $\begin{array}{c}6.75 \\
(2 \times 4 \text { MIMO })\end{array}$ \\
\hline \multirow{3}{*}{$\begin{array}{l}\text { Downlink cell spectral } \\
\text { efficiency b/s/Hz/user } \\
\text { Microcellular } 3 \mathrm{~km} / \mathrm{h} \text {, } \\
500 \mathrm{~m} \mathrm{ISD}\end{array}$} & $(2 \times 2$ MIMO) & 1.69 & 2.4 & \multirow{3}{*}{2.6} \\
\hline & $(4 \times 2$ MIMO $)$ & 1.87 & 2.6 & \\
\hline & (4x4 MIMO) & 2.67 & 3.7 & \\
\hline \multirow{2}{*}{$\begin{array}{c}\text { Uplink cell spectral } \\
\text { efficiency b/s/Hz/user } \\
\text { Microcellular } 3 \mathrm{~km} / \mathrm{h} \text {, } \\
500 \mathrm{~m} \mathrm{ISD}\end{array}$} & (1x2 MIMO) & - & 1.2 & \multirow{2}{*}{1.8} \\
\hline & (2x4 MIMO) & - & 2.0 & \\
\hline \multirow{3}{*}{$\begin{array}{l}\text { Downlink cell-edge user } \\
\text { spectral efficiency } \\
\text { b/s/Hz/user, } 5 \text { percentile, } \\
10 \text { users, } 500 \mathrm{~m} \text { ISD }\end{array}$} & $(2 \times 2$ MIMO) & 0.05 & 0.07 & \multirow{3}{*}{0.075} \\
\hline & (4x2 MIMO) & 0.06 & 0,09 & \\
\hline & (4x4 MIMO) & 0.08 & 0.12 & \\
\hline \multirow{2}{*}{$\begin{array}{l}\text { Uplink cell-edge user } \\
\text { spectral efficiency } \\
\text { b/s/Hz/user, } 5 \text { percentile, } \\
10 \text { users, 500m ISD }\end{array}$} & (1x2 MIMO) & - & 0.04 & \multirow[b]{2}{*}{0.05} \\
\hline & $(2 \times 4$ MIMO) & - & 0.07 & \\
\hline
\end{tabular}

\subsection{Physical Layer}

The basic architecture of LTE/LTE-Advanced consisting two main parts: namely EUTRAN and EPC [4]. BS and UE are the part of E-UTRAN. BS in LTE is called eNB which communicates with UEs and it can serve one or several E-UTRAN cells at a time. eNBS are directly connected with each other via a logical interface called X2 interface. On the other hand, EPC is an all-IP based core network to support the E-UTRAN to a 
seamless and uniform experience of UEs. It consists of three main network elements as MME, S-GW and P-GW. MME is the main control plane which is responsible for user mobility, intra-LTE handovers and security authentications etc. On the other hand, S-GW is mainly used for routing user data packet among different LTE nodes and performs inter-LTE handover as well as inter-3GPP mobility. The interface of MME and S-GW with eNB is referred to as S1-MME and S1-U, respectively. Finally, P-GW provides the UEs in access to a packet data network and accomplishes policy enforcement, packet filtering and perform charging functionalities.

\subsection{Transmission Schemes and Frame Structure}

LTE/LTE-Advanced has been designed as a highly flexible radio access technology which effectively supports several bandwidths from $2.5 \mathrm{MHz}$ to $20 \mathrm{MHz}$ (Release 8). Radio resources are allocated simultaneously in time and frequency domains. In the frequency domain, the total bandwidth is divided into sub-channels of $180 \mathrm{KHz}$; each one contains 12 consecutive OFDM sub-carriers with $15 \mathrm{KHz}$ equal spacing. Whereas, in time domain they are distributed into every Transmission Time Interval (TTI), each one lasting $1 \mathrm{~ms}$ and also called sub-frame. 10 consecutive subframes are grouped into frame. Moreover, each sub-frame is made of two time slots with length $0.5 \mathrm{~ms}$, corresponding to 7 or 6 OFDM symbols with normal cyclic prefix or extended cyclic prefix, respectively. Radio resource of one time slot over one sub-channel is called Physical Resource Block (PRB) which is the smallest radio resource unit that can be assigned to an UE for data transmission. However, each radio frame has two $5 \mathrm{~ms}$ halves, called acquisition channels, containing the necessary signals which are used for synchronization, providing physical cell identity (PCI) and the physical broadcast channel (PBCH). Furthermore, LTE defines a pilot signal referred to as a common reference signal (CRS), which is used for mobility measurements as well as for demodulation of the downlink control and data channels. The CRS transmission is distributed in time and frequency to enable adequate time and frequency interpolation of the channel estimates for the purpose of coherent reception of the transmitted signals.

\section{Heterogeneous Networks (HetNets)}

Heterogeneous Network (HetNets) is being considered as a most promising approach to enhance network capacity, overall performance, and to increase coverage in a cost effective way. A HetNet consists of regular macro cells transmitting typically at high power level, overlaid with low-power small cells such as Pico cell, Femto cell, Remote Radio Head (RRH), and Relay Node (RN) etc. The incorporation of such small cells allows offloading traffic from macro cell and providing better network experience by connecting UEs in small cells with low transmission power. But, this overlaying of macro cell and small cells results severe inter-cell interference in networks, in particular for celledge users of small cell. Generally, macro cells are normally placed in a cellular network attending to a prudently network plan, while the placement of low power small cells is typically based on just a knowledge of coverage issues and traffic densities (e.g. hotspots) in the network.

Different types of deployment scenarios for HetNets are already available. In multicarrier deployment, small cells utilize different carrier frequency than the macro cell. This process effectively reduces ICI but does not ensure proper spectral utilization. On the other hand, co-channel deployment is utilized by using the same carrier for both of macro cell and small cell in which the spectral efficiency is increased via spatial reuse and popular deployment approach in HetNets. Though co-channel approach ensures effective spectrum utilization but bring high ICI among the macro cell \& small cells. 


\section{Cell Range Extension (CRE)}

In LTE/LTE-Advanced cell selection is performed based on UE measurements of the received power of the cell specific reference (CRS) downlink signals. In a heterogeneous network, there have different types of base stations with diverse transmission powers, consequently different power level of CRS download signals. Therefore, cell selection based on CRS signaling power strength would not be optimal as most probably the UE will choose the higher power macro cell even if the path loss to the small is smaller. This also would not be optimal in terms of uplink coverage, downlink capacity and interferences. To optimize the uplink coverage and downlink capacity cell selection could be done by applying a cell-specific offset to the received signal power upon the estimation of the uplink path loss. This offset would somehow compensate for the transmitting power differences between the macro cell and small cell and also extend the coverage area of the small cells. This extended area is called cell range extension of small cell. However, ICI is become more severe problem at CRE area which could be mitigate by using advanced ICIC technology.

\section{Inter-Cell Interference Coordination (ICIC) in HeNets}

As mentioned earlier that inter-cell interference is the main problem towards HetNets deployment. To mitigate ICI in HetNets different types of ICI coordination techniques are available based on TDM resource partitioning, FDM based resource allocation and controlling of transmission power. In all cases tight synchronization and coordination between the interfering cells are indeed. In following subsections each of the techniques are discussed in details.

\subsection{TDM based Enhanced ICIC Techniques}

TDM based ICIC techniques are targeted to utilize full spectrum at all layers of the HetNets. The principle of TDM based enhanced ICIC (eICIC) schemes lies to restrict transmissions in certain sub-frames of the aggressor base station (macro cell) those having high probability of interference with small cell. 3GPP release-10 introduced several TDM based eICIC schemes as follows.

6.1.1. Almost Blank Subframes (ABS) based eICIC: An ABS is defined as minimum transmission subframe, where no data signal will be transmitted from the macro cell but only transmit the most critical information required for the system to provide support to legacy LTE (Release 8/9) UEs. Therefore, during ABS, the signals that are mainly sent are common reference signals (CRS) and other obligatory system information. As a result, during subframes where the macro-cell transmits ABS, the low power small cells are able to schedule UEs from a larger geographical area that otherwise would experience too high interference from the macro layer. This basically implies that using ABS at macro-cell makes possible to increase the offloading of traffic to the small cell. Moreover, this concept allows the use of higher values of CRE for the LPNs.

ABS patterns are configured in semi-statically and signaled between the macro-cell and small cell over the X2 interface. The macro-cell is supposed to act as the master that decides which subframes are to be set as ABS depending on different information regarding the cluster. Moreover, some improvements for the X2 application protocol are included in the Rellease10 specification, which makes easier the configuration of ABS muting pattern among cells. Regarding the Release 10 specification, the macro-cell supports techniques for the configuration of ABS muting patterns, being able to obtain the maximum overall system performance while considering also quality of service (QoS) requirements of individual UEs. An example of X2 signaling to configure the ABS muting pattern between the macro cell and pico cell is illustrated in fig.1. 


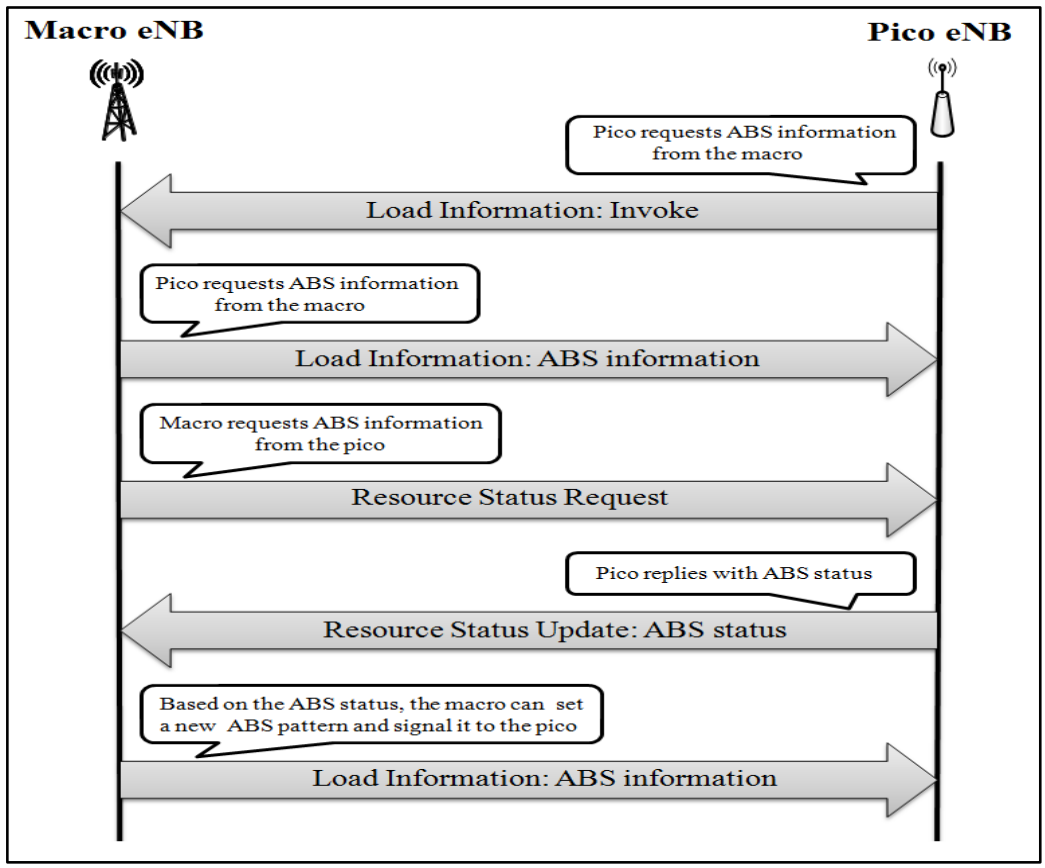

Figure 1. X2 Signaling of ABS Muting Pattern for Distributed Coordinated

As depicted in fig.1, the pico cell initiate ABS request by sending load information (LI) with information element (IE) to macro cell. The macro-cell answers by sending back another LI message with IE ABS information which includes the current ABS muting pattern used at macro-cell. In addition, the macro-cell can ask the pico cell to communicate the utilization of the allocated ABS resources by starting a resource status (RS) reporting initialization mechanism. The pico cell responds and provides the required information with a RS update with IE ABS status. Based on the ABS status from the pico cell, the macro cell has sufficient information to determine whether to use more or less subframes as ABS before deciding on a new ABS muting patter. If the macro cell makes the decision of changing the ABS muting pattern, it informs the small cells within the cluster by means of an ABS information message.

6.1.2. Reduced Power Almost Blank Subframes (RP-ABS) based eICIC: The ABS based eICIC incur valuable spectrum loss due to the requirement that the macro cell need to completely blank the transmit power on PDSCH resource elements in ABS. In this process LTE radio frames are divided into eICIC subframes and non-eICIC subframes. While the small cell could use eICIC and non-eICIC subframes, the macro cells could only use non-eICIC subframes to schedule its users. However, with reduced power almost blank subframes (RP-ABS) the macro cell doesn't completely blank the power on eICIC subframes, as it could use these subframes with reduced power to serve cell center users in the macro cell. Therefore, it's effectively utilized the entire subframe, but of course requires intelligent scheduling and coordination between the macro cell and the coordinated small cells. The amount of power reduction could be static or dynamic. With static RP-ABS, the split of eICIC subframes to non-eICIC subframes is usually fixed and the amount of power reduction could be equal to the range expansion bias and also is fixed for all eICIC subframes. Whereas in dynamic RP-ABS, the small cell scheduler must communicate with the macro cell scheduler to exchange the amount of eICIC resource blocks and the corresponding required power reduction on each eICIC resource block. Thus, the capacity gain from using reduced power subframes over almost blank subframes depends on the ratio of eICIC subframes to non-eICIC subframes within a radio frame and the intelligence of the scheduling and coordination. The macro cell 
scheduler usually allocates the RP-ABS resource blocks to cell centers, where the quality of RP-ABS resource blocks are ranked in terms of fading and power reduction then allocated to cell center to achieve the desired quality of experience determined by the scheduling goal. A typical choice for the power reduction per user is the difference in received reference powers between the interfering macro cell and the small cell. In order to have effective coordination, backhaul X2 links between the macro cell and the coordinated small cells must have relatively low latencies. As the degree of coordination of scheduling decisions nears perfection, the described dynamic RP-ABS scheme becomes almost identical to the Coordinated Scheduling feature in Coordinated Multipoint (CoMP). It is worth noting that achieving perfect coordination between macro and small cell nodes requires a centralized coordination structure (e.g. cloud RAN).

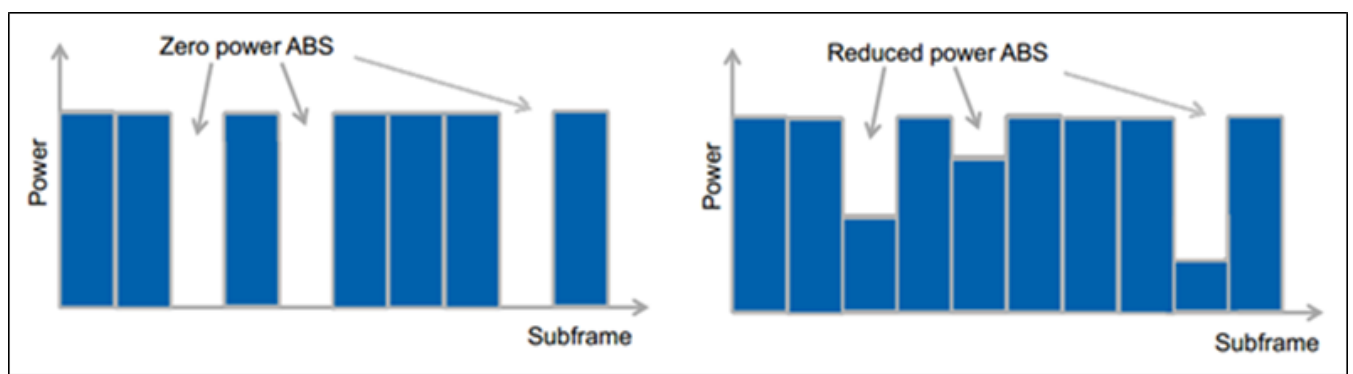

Figure 2. ABS and RP-ABS Subframes

However, with the use of ABS/RP-ABS muting patterns, more interference fluctuations occur in the network depending on whether a subframe is being used as ABS or normal. Therefore, it becomes more difficult for the cells to perform LA procedures (i.e. selection of MCS) as well as channel aware packet scheduling based on CQI feedback from UEs. As a result, the network needs to configure restricted CQI measurements for Release-10 UEs such that they can send the reports corresponding to both normal subframes and ABS to the eNB. However, Release-8/9 UEs do not support measurement restrictions, so the reported CQI is done based on an interference estimation averaged in time domain through a particular time window. Hence, these users may experience lower performance in network where eICIC is enabled. Moreover, Release10 eICIC schemes cannot able to address interference handling of CRS signals which must be transmitted to support legacy LTE (Release 8/9).

\subsection{Further Enhanced Inter-Cell interference Coordination (FeICIC)}

As stated at section 6.1.2, eICIC cannot handle CRS interference of neighboring cells which could be detrimental for the demodulation of various data and control channels. Based on the reference signal frequency hopping CRS of neighboring cells are classified as colliding and non-colliding CRS. While non-colliding CRS interferes with data and control channel, colliding CRS interferes with other CRS signal which lead poor channel estimation. However, 3GPP Release 11 introduced FeICIC which can able to manage CRS interference solution based on the transmitter and receiver actions. The techniques are described as follows.

6.2.1. Transmitter based FeICIC: The transmitter based FeICIC approach rely on muting PDSCH resource elements within a small cell ABS that experiences strong interference from macro CRS. To compensate for the minor PDSCH resource loss a rate matching is then applied by changing the rate of coding and rate matching. A transmitter based FeICIC approach is illustrated in fig. 3 for the non-colliding CRS case. In transmitter based FeICIC, the transmitter mutes PDSCH resource elements vulnerable to strong CRS interference. However, in order to support legacy UEs, this type of muting is 
not currently feasible for the downlink control channels, as PDCCH resource could not be muted or rate matched.

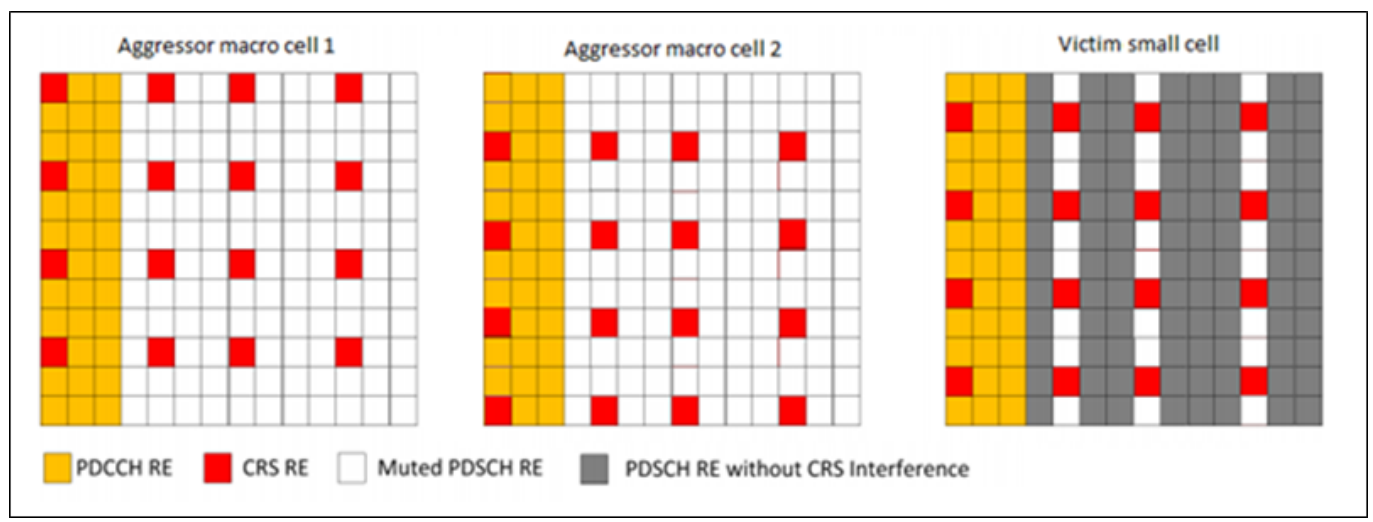

Figure 3. Transmit-based FeICIC CRS Interference Avoidance

6.2.2. Receiver based FeICIC: The receiver based FeICIC approach eliminate the dominant CRS interference based on the estimation at UE receiver end. In the case of non-colliding CRS, this technique is more effective since the UE is able to distinguish the strong interferers then select the dominant interferer. Once the UE estimates the CRS of the strongest interferer, it can subtract that from the received signal. It is worth noting that the UE could perform this process for multiple interferers, but it would require an iterative process to subtract each interfering signal. However, it is not advisable to cancel the interference from weak interferers, as it would difficult to estimate such signals; weak CRS interference does not significantly contribute to the overall interference, so there is little harm in neglecting it at the receiver. However, in the case of colliding CRS, receiver based elimination is not effective since the UE may find some trouble estimating the interfering reference signals as the UE requires information from the network regarding which cells are suitable for CRS cancellation at the UE.

\subsection{FDM Based ICIC}

The basic principle of FDM based ICIC is to divide the available spectrum into parts and the interfering cells will use different frequency spectrum. However, 3GPP release 10 introduced a unique feature called Carrier Aggregation (CA) which enables multicarrier scheduling in LTE-Advanced system. In this section, the introduction of CA and ICIC in HetNets using CA are discussed.

6.3.1. Carrier Aggregation (CA): Carrier aggregation (CA) is one of the key features of LTE-Advanced (Release 10). LTE-A is designed to meet the peak data rates required by IMT-Advanced: $1 \mathrm{Gbps}$ for the downlink and $500 \mathrm{Mbps}$ for the uplink. This requires UE access to a total bandwidth up to $100 \mathrm{MHz}$. Since the maximum supported bandwidth in LTE is $20 \mathrm{MHz}$, bandwidth is expanded through aggregating up to five component carriers (CCs). Moreover, CA is designed to be backward compatible, meaning that both LTE (Release 8/9) and LTE-Advanced UEs can be supported in the same CC deployed by the Release 10 eNodeB (eNB). Each CC should inherit the bandwidth configurations of the Release 8 carriers, for example, it should be in the size of $1.4,3,5,10,15$, or $20 \mathrm{MHz}$ which are the typical LTE carrier sizes. Moreover, different carriers may be in different sizes, giving operators flexibility in forming the required bandwidth subject to the available spectrum constraints. Such spectrum compatibility is of critical importance for a smooth, low-cost transition to LTE-Advanced capabilities within the network. 
6.3.2. CA based ICIC in HetNets: CA based interference management lies on the divide of the full spectrum into two parts, name primary cell (PCell) and secondary cell (SCell); illustrates in fig.4. Different deployment scenario is presented to design HetNets by means of CA which can successfully mitigate the interference effect. Based on the centralized architecture, the macro cell with full coverage area could serve as PCell and small cells would serve as SCell. Macro cell is responsible to provide system information, control signaling and allowed to bandwidth limited data transmission, while small cells would serve local high rate data transmission. In case of dedicated carriers for macro cell and small cell this CA technique would be more beneficial.

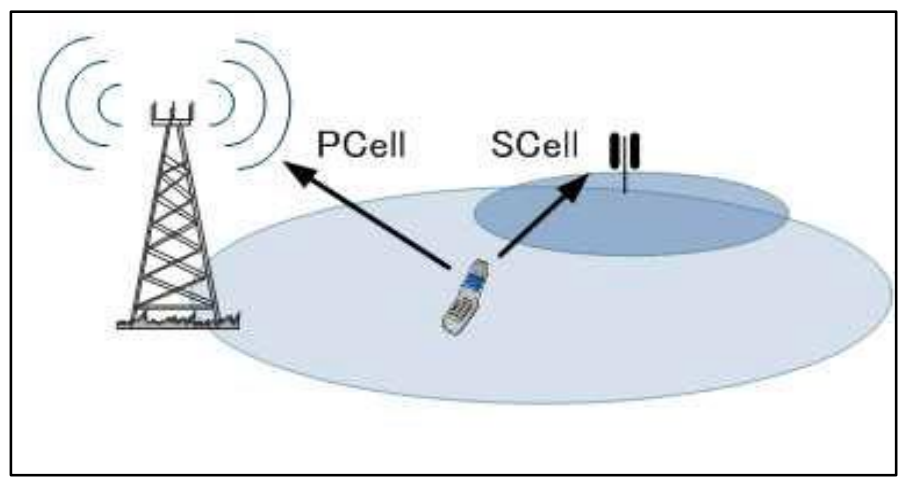

Figure 4. LTE-A HetNet Multi-site Carrier Aggregation

Nevertheless, there are also drawbacks of this functionality. First of all, requirement of a centralized architecture; that is less supportive in LTE-Advanced standard system. Furthermore, multiple Timing Advance (TA) supporting CA capable terminals are required; this will basically require two transmitters in the terminal which is a challenging step in implementation.

In an alternative deployment, not necessarily based on a centralized architecture, macro cell and the small cells might operate with own control signaling on both frequency layers. Such deployment will require advanced interference management. In this case, both of macro cell and small cell will allocate different frequency layers for their respective PCell and SCell. The SCell might not transmit any data or may transmit data with lower power to avoid interfering with the PCell of the other layer. Depending on the implementation of the radio resource management and scheduling algorithm, UEs close to the base station can be scheduled with low power SCell allocations, while cell edge users are allocated using the PCell. Power variations of the SCell to a single UE can only be semi-static, since the power difference to the reference symbols needs to be known by the UE (signaled on slow basis by RRC in LTE) for amplitude modulation. For full protection of the control region, PDCCH Cross Carrier Scheduling from the Pcell can be used. If the Scell's PDCCH is not used at all, interference on the PCell's PDCCH is minimized. Spectrum efficiency also can be increased as frequencies are available at macro and small cells. Figure 5 demonstrates the cross-carrier scheduling of primary carrier and secondary carrier in CA based HetNets. 


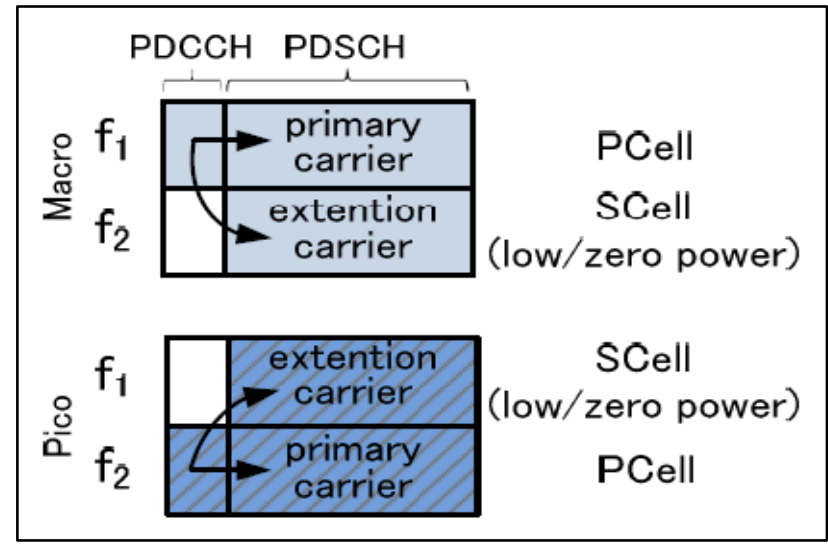

Figure 5: LTE-A HetNet using CA based Cross-carrier Scheduling

However, this CA based ICIC has some benefits compared to a TDM based ICIC using almost blank subframes as the Release 8/9 legacy terminals are always supported on the PCell without performance degradation. Although at least a certain penetration of CA capable UEs is required. Overall, the level of complexity for network integration, to support user mobility, the signaling and measurement overhead is likely to be higher in a co-channel deployment using eICIC. However, one issue of this approach is that eventually the macro cell might run out of $\mathrm{PDCCH}$ resources due to its use of cross carrier scheduling. PCell and SCell resource allocations have to be signaled separately, potentially doubling the load on the downlink control channel. On top of this, additional PDCCH capacity could be required to support future Multi-User MIMO, where resources are allocated to multiple UEs in the spatial domain.

To reduce the PDCCH load 3GPP Release 11 introduces a new downlink control channel concept called Enhanced Physical Downlink Control Channel (ePDCCH). The ePDCCH is a new downlink control channel reusing physical resource block (PRB) formerly used for PDSCH transmission. This concept provides various benefits over earlier two techniques. In addition, the general increase of control channel flexibility, ePDCCH also effectively supports the HetNet scenario as described above. The ePDCCH could provide downlink control signaling resources within each SCell without the use of cross carrier scheduling and without interfering with the PDCCH of the other layer's PCell. The reliability of the ePDCCH could be increased in case frequency domain interference coordination is applied between sites. In that case SCell's ePDCCH resource blocks are not scheduled in the other layer's PCell. Figure 6(b) illustrates this principle that can be applied in case ePDCCH performance is interference limited. However, proper coordination between the macro cell and small cells also required here.

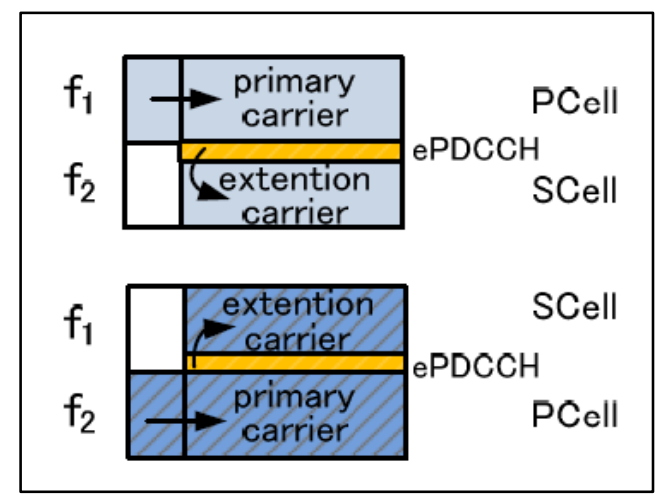

Figure 6(a): CA based HetNet using PDCCH

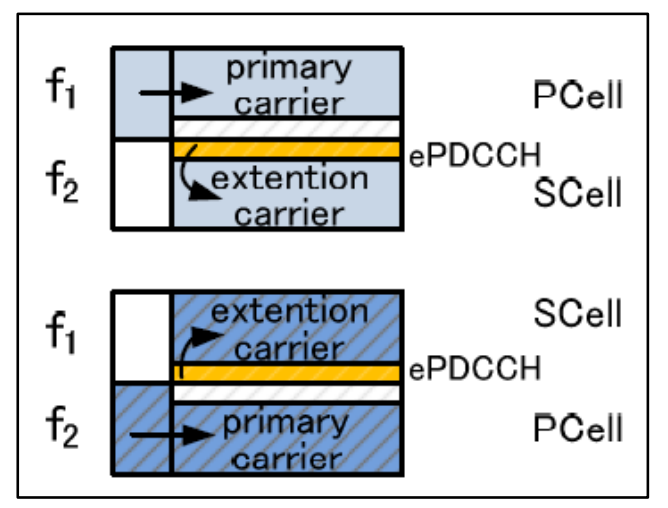

Figure 6(b): CA based HetNets using ePDCCH with ICIC 


\subsection{Coordinated Multi-Point (CoMP) based ICIC}

The fundamental principle of CoMP is utilized multiple transmit and receive antennas from multiple antenna site locations which may or may not belong to the same physical cell. CoMP mainly has been targeted to improve cell-edge UE experience, but regardless the location it use to enhance system throughput of UEs those experience strong signals of different cells. CoMP mainly categorized as inter-site CoMP in which the coordination is performed between cells located at separated geographical areas, and intra-site CoMP which enables the coordination between sectors of the same eNB. Also, in downlink scenarios, CoMP transmissions are classified as Coordinated Beam-Switching (CBSCoMP), Coordinated Scheduling (CS-CoMP), Joint Transmission (JT-CoMP) and Dynamic Point Selection (DPS-CoMP). In first two cases UEs data would be available only at serving cell but coordination is necessary between all of the interfering cells. Whereas in later two cases, UEs data might be available at more than one cells; by default at all coordinating cells, but transmission may be occurred at one or more cells. In addition, depending on coordination architecture CoMP are also divided into two main schemes, namely Central CoMP and Distributed CoMP. A detail survey report in CoMP transmission is presented in [25].

In [26]-[31], several techniques available for CoMP technology but all the approaches require to share some scheduling information regarding the UEs data at coordinating cells. This sharing results increased complexity and signaling overhead on X2 interface. However, in downlink CoMP, UEs report Channel State Information (CSI) during uplink transmission to the cells based on the experienced SINR of respective eNBs. In CoMP system, to indicate the possible channel states in both transmitter and receiver a set of predefined precoding matrices is used. The Precoding Matrix Index (PMI), which is defined as the index to the preferred matrix within in a codebook matrix, is reported by UE together with Channel Quality Indicator (CQI) and MIMO Rank Indicator (RI). CQI and PMI are parameters determined by UE based on pilot measurements and UEs send to their serving BS. Moreover, RI indicates the number of data streams to be transmitted in parallel for the next transmission over the MIMO channel. These parameters are transmitted in a quantized manner to eNB targeting to reduce signaling overhead. By coordinating and combining signals from multiple antennas, CoMP makes it possible for UEs to enjoy consistent performance and quality when they access and share videos, photos and other high bandwidth services whether they are close to the center of an LTEA cell or at its outer edges.

\section{Conclusion}

In this article, an extensive survey on inter-cell interference management in LTEAdvanced HetNets has been performed. The survey has covered all possible techniques of ICIC in LTE-Advanced HetNets. It has clearly identified the benefits as well as drawbacks of each the techniques with all possible modifications. The TDM based and CA based advanced ICIC mechanisms, which examined in this survey, provide a general framework of solutions that can handle efficiently inter-cell interference as one of the major issues in the wireless heterogeneous networks.

The TDM based ICIC mechanisms have been thoroughly investigated and categorized based on a variety of key features. As discussed, co-channel deployment could ensure proper utilization of the available spectrum but could not able to manage ICIC in a greater degree. However, the introduction of reduced power almost blank subframes effectively overcome PDSCH and PDCCH interference of aggressor cells, but fallen short to handle CRS interference. Whereas, based on the transmitter/receiver action FeICIC is considered as a promising solution to CRS interference cancellation/avoidance in HetNets. In all of the cases tight and efficient synchronization between the interfering cells are indeed. 
On the other hand, carrier aggregation has been considered as a most promising solution to deploy LTE-Advanced HetNets. CA could effectively manage the ICIC by assigning different component carriers to different cells, but this technique could not ensure proper spectrum utilization. However, release 10 and 11 introduced cross-carrier scheduling and enhanced control channel ePDCCH, respectively, which ensure proper bandwidth utilization as well as effective ICIC in HetNets. CA based ICIC also offers some benefits over TDM based ICIC in case to support legacy LTE UEs. Finally, CoMP has been further explored as a complementary scheme for ICIC methods by characterizing their partially common features. As described, CoMP provides an advanced coordination framework suitable for high order MIMO-OFDMA systems like LTE-Advanced, but required low latency $\mathrm{X} 2$ interference as well as strict synchronization among the coordinating cells.

\section{References}

[1] Ericsson White Paper, "Differentiated mobile broadband" (2011).

[2] Cisco White Paper, "Cisco visual networking index: Global mobile data traffic forecast update", (2012).

[3] M. Rumney, "LTE and the Evolution to 4G Wireless: Design and Measurement Challenges", Copyright Agilent Technologies, (2013).

[4] A. Toskala and H. Holma, "LTE-Advanced 3GPP Solution for IMT-Advanced", John Wiley \& Sons Ltd., (2012).

[5] 3GPP TR36.814, "Further advancements for E-UTRA physical layer aspects," Technical report, 3GPP, (2010).

[6] 3GPP TR36.913, "Requirements for further advancements for E-UTRA," Technical report, 3GPP, (2012).

[7] A. Damnjanovic, J. Montojo, Y. Wei, T. Luo, M. Vajapeyam, T. Yoo, O. Song and D. Malladi, "A Survey on 3GPP Heterogeneous Networks", IEEE Wireless Communications, vol. 18, no. 3, (2011), pp.10-21

[8] A. Ghosh, N. Mangalvedhe, R. Ratasuk, B. Mondal, M. Cudak, E. Visotsky, T. Thomas, J. Andrews, P. Xia, H. S. Jo, H. Dhillon and T. Novlan, "Heterogeneous cellular networks: From theory to practice", IEEE Communication Magazine, vol. 500, no. 6, (2012), pp. 54-64

[9] N. Saquib, E. Hossa and Dong In Kim, "Fractional Frequency Reuse for Interference Management in LTE-Advanced HetNets", IEEE Wireless Communications, (2013).

[10] Y. Wang and K. Pedersen, "Performance Analysis of Enhanced Inter-cell Interference Coordination in LTE-Advanced Heterogeneous Networks", IEEE Vehicular Technology Conference, (2012).

[11] D. Lopez-Perez, I. Guvenc, G. de la Roche, M. Kountouris, T. Q. S.Quek, and J. Zhang, "Enhanced intercell interference coordination challenges in heterogeneous networks", IEEE Wireless Communication Magazine, vol. 18, no. 3, (2011), pp. 22-30.

[12] Y. Chen, X.Fang and B. Huang, "Joint ABS Power and Resource Allocations for eICIC in Heterogeneous Networks", 6th IEEE International Workshop on Signal Design and Its Applications in Communications, (2013).

[13] S. N. S. Kaimalettu, S. R. Yerrapareddy, K. Milleth and N. Akhtar, "On Interference Management Based on Subframe Blanking in Heterogeneous LTE networks," IEEE Conference on Communication Systems and Networks, (2013), pp.1-7.

[14] M. Zeng, X.Chen, X. Zhang, L. Sang and D. Yang, "A Novel Dynamic Interference Coordination Scheme in Macrocell-Picocell Heterogeneous Networks", 77th IEEE Vehicular Technology Conference, (2013), pp. 1-5.

[15] S. Vasudevan, R. N. Pupala and K. Sivanesan, "Dynamic eICIC - A Proactive Strategy for Improving Spectral Efficiencies of Heterogeneous LTE Cellular Networks by Leveraging User Mobility and Traffic Dynamics", IEEE Transactions on Wireless Communications, vol.12, no. 10, (2013), pp. 49564969.

[16] M. Y. Umair, Y. Dongkai, D. Xiao and F. Fanny, "Identification of Interferers in HetNet in LTE-A Systems Based on FeICIC with Cell Range Expansion," IEEE Conference on Information and Communication Technology (ICoICT), (2013), pp. 198-201.

[17] E. Pateromichelakis, M. Shariat, A. Quddus and Rahim Tafazolli, "On the Evolution of Multi-Cell Scheduling in 3GPP LTE / LTE-A", IEEE Communication Surveys \& Tutorials, (2012).

[18] L. Gao, "Heterogeneous Networks - Theory and Standardization in LTE", Tutorial Presentation, IEEE WCNC, (2013). 
[19] Q. Li, R. Q. Hu, Y. Qian and G. Wu, "Cooperative Communications For Wireless Networks: Techniques and Applications in LTE-Advanced Systems", IEEE Wireless Communications, (2012).

[20] R. Irmer, H. Droste, P. Marsch, M. Grieger, G. Fettweis, S. Brueck, H-P. Mayer, L. Thiele and V. Jungnickel, "Coordinated Multipoint: Concepts, Performance, and Field Trial Results", IEEE Communication Magazine, (2011), pp. 102-111.

[21] D. Lee, H. Seo, B. Clerckx, E. Hardouin, D. Mazzarese, S. Nagata and K. Sayana, "Coordinated Multipoint Transmission and Reception in LTE-Advanced: Deployment Scenarios and Operational Challenges", IEEE Communication Magazine, (2012), pp. 148-155.

[22] M. S. Ali, "On the Evaluation of Coordinated Multi-Point (CoMP) transmission in LTE-A," International Journal of Future Generation Communication \& Networking, vol. 7, no. 4, (2014).

[23] H. Ekstrom, "QoS Control in the 3GPP Evolved Packet System", IEEE Communication Magazines, vol. 47, (2009), pp. 76-83.

[24] T. E. Kolding, "QoS-Aware Proportional Fair Packet Scheduling with Required Activity Detection," VTC Fall, (2006).

[25] Md. S. Ali, "On the Evaluation of Coordinated Multi-Point (CoMP) transmission in LTE-A," International Journal of Future Generation Communication and Networking (Published by SERSC), vol. 7, no. 4, (2014), pp. 91-102

[26] D. Gesbert, S. Hanly, H. Huang, S. Shamai, O. Simeone and W. Yu, "Multi-cell MIMO Cooperative Networks: A New Look at Interference", IEEE Journal of Selected areas of Communications, vol. 28, no. 9, (2010), pp. 1380-1408

[27] 3GPP R1-110461, "Baseline schemes and focus of CoMP studies", Ericson TSG-RANWG1, no. 63, (2011).

[28] 3GPP, "Mobile Broadband Innovation Path to 4G: Release 9, 10 and Beyond", TR, (2010).

[29] D. López-Pérez, I. Güvenç, G. Roche, M. Kountouris and T. Quek, J Zhang, "Enhanced Inter-Cell Interference Coordination Challenges in Heterogeneous Networks", IEEE Wireless Communication, vol. 18, no. 3, (2011), pp. 22-30

[30] Q. Li, R. Q. Hu, Y. Qian and G. Wu, "Cooperative Communications For Wireless Networks: Techniques and Applications in LTE-Advanced Systems", IEEE Wireless Comm., (2012), pp. 22-29.

[31] R. Irmer, H. Droste, P. Marsch, M. Grieger, G. Fettweis, S. Brueck, H-P. Mayer, L. Thiele and V. Jungnickel, "Coordinated Multipoint: Concepts, Performance, and Field Trial Results", IEEE Communication Magazine, (2011), pp. 102-111.

\begin{abstract}
Author
Md. Shipon Ali achieved his B.Sc. Engineering degree in Electronics \& Communication Engineering from Khulna University, Bangladesh in 2009. He achieved 'University Gold Medal' from Khulna University for securing first position with distinction by obtaining the highest CGPA in Bachelor Examinations among all disciplines under School of Science, Engineering and Technology in the session 20072008. Now, he is working as a 'Senior Engineer' in a multi-national telecom operator in Bangladesh. His research interests are dynamic radio resource management \& interference management in LTE-A HetNets, wireless network, source/channel coding, OCDMA, advanced DSP and fiber optics communication.
\end{abstract}


International Journal of Future Generation Communication and Networking Vol. 8, No. 1 (2015) 